\title{
ERRATUM
}

\section{Genetic Otx2 mis-localization delays critical period plasticity across brain regions}

HHC Lee, C Bernard, Z Ye, D Acampora, A Simeone, A Prochiantz, AA Di Nardo and TK Hensch

Molecular Psychiatry (2017) 22, 785; doi:10.1038/mp.2017.83; published online 4 April 2017

Correction to: Molecular Psychiatry (2017) 22, 680-688; doi:10.1038/mp.2017.1; published online 14 February 2017
The Supplementary Materials and Figure legends were omitted from this paper when it published. The missing data accompanies this erratum online. The publisher regrets the error. 